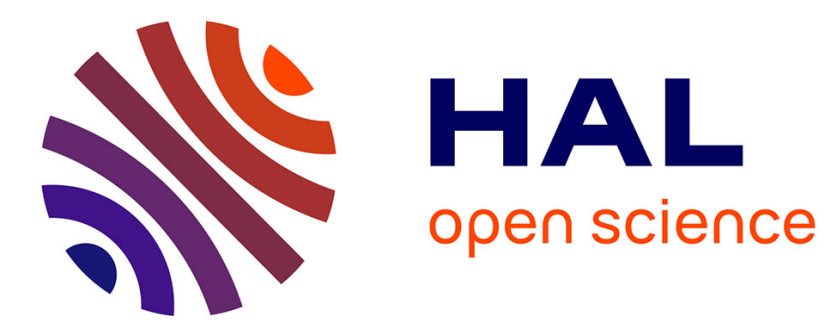

\title{
Analysis of resonant pull-in of micro-electromechanical oscillators
}

Jérôme Juillard

\section{To cite this version:}

Jérôme Juillard. Analysis of resonant pull-in of micro-electromechanical oscillators. Journal of Sound and Vibration, 2015, 350, pp.123-139. 10.1016/j.jsv.2015.03.056 . hal-01235573

HAL Id: hal-01235573

https://hal-centralesupelec.archives-ouvertes.fr/hal-01235573

Submitted on 30 Nov 2015

HAL is a multi-disciplinary open access archive for the deposit and dissemination of scientific research documents, whether they are published or not. The documents may come from teaching and research institutions in France or abroad, or from public or private research centers.
L'archive ouverte pluridisciplinaire HAL, est destinée au dépôt et à la diffusion de documents scientifiques de niveau recherche, publiés ou non, émanant des établissements d'enseignement et de recherche français ou étrangers, des laboratoires publics ou privés. 


\title{
Analysis of resonant pull-in of micro-electromechanical oscillators
}

\author{
Jérôme Juillard, GEEPS/ECO2/SE, CentraleSupélec, France
}

\begin{abstract}
In this paper, the equations governing the pull-in of electrostatic MEMS (microelectromechanical systems) oscillators are established and analyzed. This phenomenon defines the maximal oscillation amplitude that can be obtained without incurring instability and, hence, an upper limit to the performance of a given device. The proposed approach makes it possible to accurately predict pull-in behaviour from the purely resonant case, in which the electrostatic bias is very small, to the static case. The method is first exposed in the case of a parallel-plate resonator and the influence of the excitation waveform on the resonant pull-in characteristics is assessed. It is then extended to the more complex case of clamped-clamped and cantilever beams. The results are validated by comparison with transient simulations.
\end{abstract}

\section{Introduction}

The static pull-in of capacitive MEMS structures is a well-studied phenomenon, theoretically and experimentally [1-2]. In particular, it is well-known that a voltage-controlled parallel-plate capacitor may not be displaced by electrostatic forces to a position exceeding one third of the gap without incurring instability. The validity of this result is limited to the case when the bias voltage applied to the capacitor is increased quasi-statically or when the structure is critically damped. Several works have been concerned with establishing similar results to the case when the structure is under-damped, mostly when the voltage is applied as a step [3-5] or when it is a superposition of a DC and of an AC component [6-8]. The latter case is of great importance for resonant sensing applications, in which the resonance frequency of a structure is measured to track changes in a physical quantity. In this context, it is important to determine the largest stable oscillation amplitude since it determines the maximal magnitude of the detected signal, and, consequently, sets the constraints on the design of the associated electronics of the MEMS/electronics interconnection scheme [9-10].
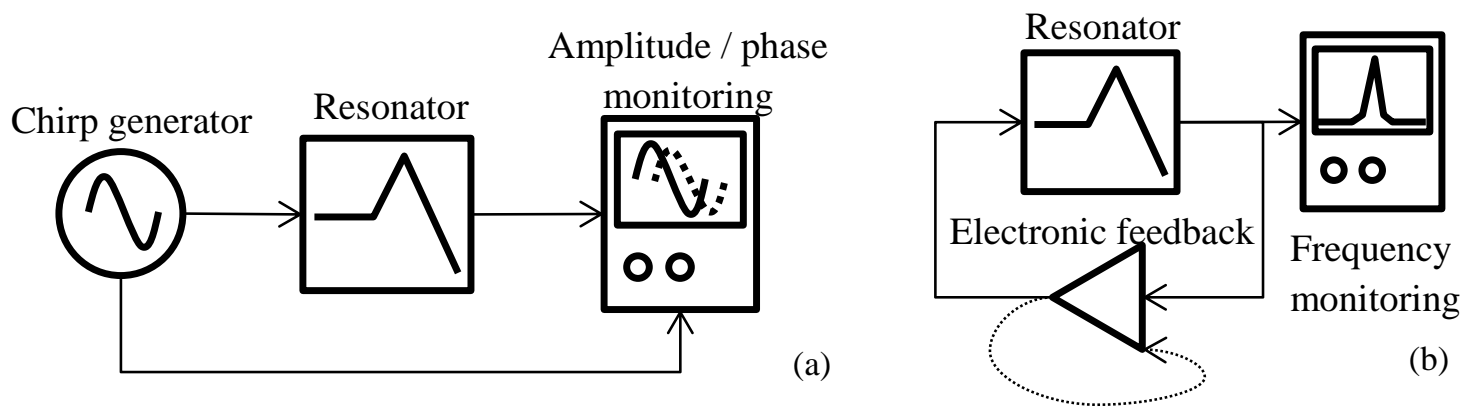

Fig.1 - Open-loop (a) and closed-loop (b) techniques for resonance frequency measurements. In the open-loop technique, the resonator is typically excited with a chirp. The amplitude and phase response of the system are obtained by demodulating the excitation and the resonator output. In the closed-loop technique, the resonator is excited with the signal output by an electronic circuit enforcing some self-oscillation conditions in the loop. The feedback electronics may include a phase-locked loop in order to guarantee a specific input-output phase relationship, as symbolized by the dotted arrow, or may have a purely feed-forward architecture.

The measurement of the resonance frequency of a MEMS structure can be performed using open-loop or closed-loop techniques (Fig. 1). This paper is dedicated to the latter case, in which feedback electronics sense the position of the resonator and actuate it close to its resonance frequency by enforcing a set of so-called "self-oscillation" conditions (Barkhausen criterion) [11]. Although the electrostatic closed-loop scheme is ubiquitous [12-17], its stability has received little attention so far. Most papers dedicated to resonant pull-in are concerned with open-loop techniques [6-8], in which the excitation frequency is controlled and the amplitude and phase of the displacement are free to vary. Only a handful of papers [18-20] are dedicated to resonant pull-in in closed-loop, in which the phase is 
set and the amplitude and frequency vary. Of these, [18] is dedicated to the case when the resonator is a parallel-plate capacitor actuated with a square-wave voltage and is only valid when the DC bias applied to the structure is very small compared to the static pull-in voltage. These three limitations (geometry, waveform and bias voltage) prevent using the results from [18] in the majority of resonant MEMS/NEMS applications. Such applications are most often based on sine-wave actuation, operate at finite bias voltage and have flexible moving parts, very far from the plane-capacitance hypothesis. In [19], the framework of [18] is expanded to account for other nonlinearities (caused by strainstiffening) and the study is made for large DC bias. However, as shown in the present paper, the approach used by the authors of [19] does not take into account the interdependence of the static component of the displacement and the oscillation amplitude. Consequently, this approach leads to qualitatively and quantitatively inaccurate results, especially at large DC bias. In [20], resonators of various shapes (parallel-plate, bridge or cantilever beams) with different types of actuation waveforms (square-wave, sine-wave, pulses) and large DC bias are considered. However, the study is limited to the case of symmetric double-sided actuation, in which the static component of the displacement is equal to zero. In an electrostatic MEMS resonator, asymmetry may result from several causes, the most common being that a single electrode is used to actuate the resonator and sense its position; in the present paper, we show that, in such asymmetric cases, the static component of the displacement (resulting from the asymmetry) plays a key role in the pull-in phenomenon.

In this paper, the case of a resonator (parallel-plate, bridge, or cantilever) actuated from only one side is considered and the influence of the excitation waveform on the resonant pull-in amplitude is studied. The main contributions of the paper are that

- a general methodology, based on harmonic balancing, for deriving the resonant pull-in condition in asymmetric electrostatic resonators is derived.

- this theory is valid from zero DC bias (purely resonant pull-in, as in [18]) to large DC bias (static, non-resonant pull-in).

- the resonant pull-in conditions are given in terms of oscillation amplitude, oscillation frequency and static component of displacement for common resonator geometries and excitation waveforms.

- voltage pull-in conditions are also established for these geometries and waveforms, in terms of maximal actuation voltage versus bias voltage.

- it is shown that, as in the case of double-sided actuation [20], the greater travel range for a given DC bias is obtained with pulse-actuation, regardless of the geometry of the resonator.

The outline of the paper is the following. In section 2, the framework and the model governing the transient dynamics of a parallel-plate resonator are described. In section 3, the equations governing the averaged dynamics of the model are established, based on a describing function approach [11]. Section 4 presents the method for deriving the resonant pull-in characteristics from the averaged dynamics. The analysis is based on an extension of one of the stability criteria presented in [11] to the case when the displacement of the structure has an amplitude-dependent DC component. This is illustrated in the case of square-wave actuation and show that the method proposed in [19] for determining resonant pull-in is faulty in the case of a finite DC bias. Section 5 discusses the limits of this work and its extension to more complex cases, in particular to the case of clamped-clamped and cantilever beams. Section 6 contains some concluding remarks.

\section{Framework}

We consider the one-sided closed-loop electrostatic actuation of a parallel-plate resonator with stiffness $K$, mass $M$, natural pulsation $\omega_{0}=\sqrt{K / M}$ and quality factor $Q$. An electrode with surface area $S_{e}$ is situated across a gap $G_{0}$ from the resonator, supposed to be very small with respect to the lateral dimensions of the electrode. A voltage $V(t)=V_{b}(1+v(t))$ is applied across the gap and exerting an electrostatic force on the resonator and setting it into motion. Throughout the paper, the 
actuation voltage is assumed to be very small with respect to the bias voltage (i.e. $v \ll 1$ ). The influence of this hypothesis is illustrated in section 4.3.

The position of the resonator as it moves across the gap is given by $G(t)=G_{0} a(t)$, so that $a=1$ means that the structure touches the opposite electrode (Fig. 2).

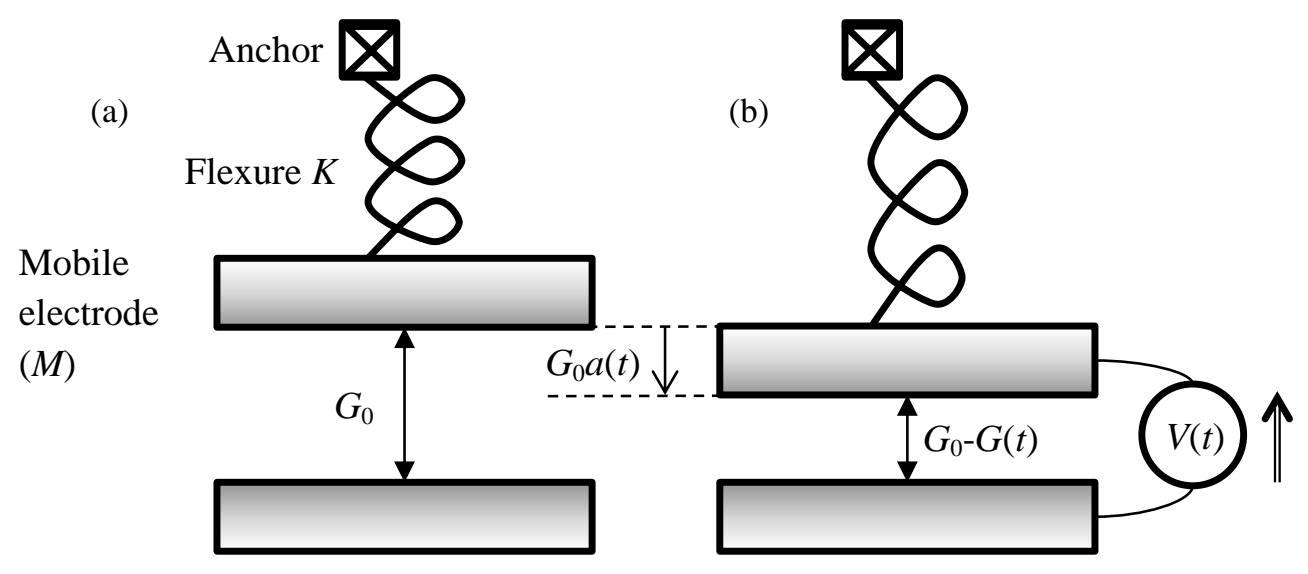

Fig. 2 - Parallel-plate resonator with no voltage applied ( $a$ ) and in a deflected position (b).

Letting a dot ( $\operatorname{as}$ in $\dot{a}$ ) denote differentiation with respect to $\tilde{t}=\omega_{0} t$, and defining the non-dimensional bias voltage as:

$\delta=\frac{\varepsilon_{0} S_{e} V_{b}^{2}}{2 K G_{0}^{3}}$,

where $\varepsilon_{0}$ is the permittivity of vacuum, the dynamics of the resonator are governed by:

$\ddot{a}+\frac{\dot{a}}{Q}+a=\delta\left(\frac{1+v}{1-a}\right)^{2}$

If $v<<1$, as assumed, the dynamics simplify to:

$\ddot{a}+\frac{\dot{a}}{Q}+a=\delta \frac{1+2 v}{(1-a)^{2}} \triangleq \delta(1+2 v) N(a)$,

where $N(a)$ represents the dependence of the electrostatic force with respect to $a$. Throughout the rest of this paper, the tilde is dropped and $t$ is used to describe non-dimensional time.

It is assumed that $a=A_{0}+A \sin (\omega t)$ and that the (non-dimensional) actuation voltage $v$ is in quadrature with $a$ so that an oscillator loop is formed. As we will show in sections 3 and 4 , the static component of the displacement, $A_{0}$, plays an important role in the stability of the oscillator. This makes the analysis of single-sided resonators more involved than that of double-sided resonators [20].

The following three waveforms are considered, which can easily be generated in closed loop when $a$ or $\dot{a}$ is measured:

- Square-wave: $v=v_{0} \operatorname{sign}(\cos (\omega t))$.

- Sine-wave: $v=v_{0} \cos (\omega t)$. 
- Pulses: $v=v_{0} \Delta(a, \dot{a}, \tau)$, where $\Delta(a, \dot{a}, \tau)$ is equal to $\operatorname{sign}(\dot{a})$ for a duration $\tau<<2 \pi$ when $a$ crosses the threshold $A_{0}$, and is equal to 0 otherwise.

(a)

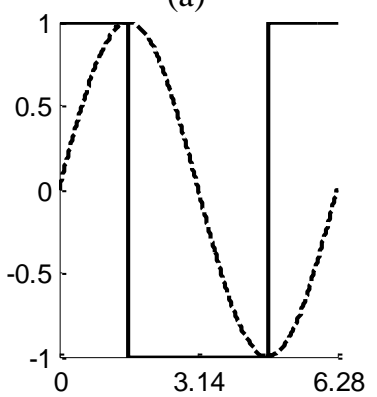

(b)

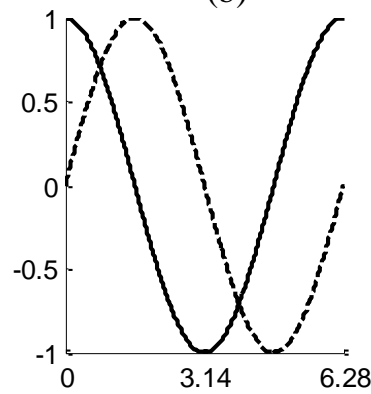

(c)

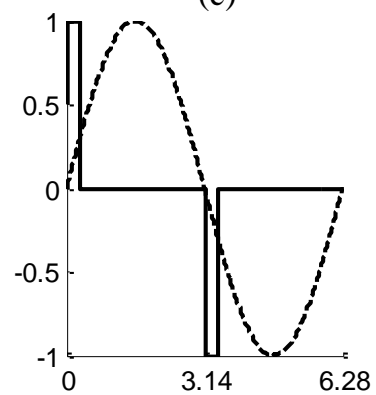

Fig. 3 - From left to right: square (a), sinusoidal $(b)$ and pulse (c) waveforms (continuous line), for $v_{0}=1$. The dashed line represents $\sin (t)$.

The three waveforms, represented in Fig. 3, can be used to make the resonator oscillate, because the first harmonic of $v$ is, by design, in quadrature with $a$ (the out-of-quadrature case is addressed in section 5). Square and sine waveforms are the most commonly used. Pulsed actuation was introduced in [21] and studied in [20-22]. Pulses can be efficiently generated by high-pass filtering the sign of the detected signal, as described in [26]. Among the advantages of pulse-actuation is the possibility to reach very large oscillation amplitude regimes (hence, large signal-to-noise ratios) [22]. Also, in capacitive MEMS applications, the detected signal is often a superposition of the useful, motional signal (proportional to $a$ ) and of a parasitic signal (proportional to $v$ ) due to capacitive feedthrough. This feedthrough phenomenon makes it difficult to guarantee quadrature between $v$ and $a$ when sinewave and square-wave actuation are used. When pulsed actuation is used, this problem is irrelevant, because the sign of $a+\lambda v$ (where $\lambda>0$ represents an unknown amount of capacitive feedthrough) is the same as the sign of $a$, no matter how large $\lambda v$ may be [22]. This simplifies the design of the oscillator [26] and can also be put to use for performing open-loop characterization of the resonator. However, the pulsed-actuation technique is practically limited by the amount of energy that can be supplied to the resonator at each period (the shorter the pulse, the larger $v_{0}$ must be), and by the fact that it may become difficult to generate well-defined pulses at very high frequencies (say, greater than a few tens of $\mathrm{MHz}$ ).

The static pull-in position $a_{s p i}$ is defined as the position for which the equilibrium of (3) becomes unstable, when $v$ and all time derivatives equal 0. It is well-known [1] that

$a_{s p i}=\frac{1}{3}(4)$

and that this position is reached for:

$$
\delta \stackrel{\Delta}{=} \delta_{s p i}=\frac{4}{27} .
$$

\section{Steady-state dynamics}

\section{Determination of the steady-state regime}

Describing function analysis [11] can be applied to (3) to determine the existence of steady-state solutions. We define: 


$$
f_{D C}=\frac{\omega}{2 \pi} \int_{0}^{\frac{2 \pi}{\omega}} N(a) d t, f_{\text {sin }}=\frac{\omega}{\pi} \int_{0}^{\frac{2 \pi}{\omega}} N(a) \sin (\omega t) d t, f_{\text {cos }}=\frac{\omega}{\pi} \int_{0}^{\frac{2 \pi}{\omega}} \frac{v(t)}{v_{0}} N(a) \cos (\omega t) d t
$$

These quantities have analytical expressions that are summarized in table 1, where the following notation is used:

$$
R=\frac{A}{1-A_{0}}
$$

Although it is not mandatory to perform this change of variables to analyze the system, the use of the "reduced amplitude" $R$ proves to be very convenient, mostly because $R$ varies between 0 and 1 , whereas $A$ varies between 0 and $1-A_{0}$, which is quantity that depends on the bias level $\delta$ applied to

\begin{tabular}{|c|c|c|}
\hline$f_{D C}$ & $f_{\text {sin }}$ & \\
\hline$\frac{1}{\left(1-A_{0}\right)^{2}\left(1-R^{2}\right)^{3 / 2}}$ & $\frac{2 R}{\left(1-A_{0}\right)^{2}\left(1-R^{2}\right)^{3 / 2}}$ & \\
\hline$f_{\cos }($ square wave $)$ & $f_{\cos }$ (sine wave) & $f_{\text {cos }}$ (pulses) \\
\hline 4 & 1 & $2 \omega \tau$ \\
\hline$\overline{\pi\left(1-A_{0}\right)^{2}\left(1-R^{2}\right)}$ & $\overline{\left(1-A_{0}\right)^{2} R^{2}} \overline{\left(1-R^{2}\right)^{1 / 2}}-1$ & $\overline{\pi\left(1-A_{0}\right)^{2}}$ \\
\hline
\end{tabular}
the system.

Table 1 - Expressions of $f_{D C}, f_{\mathrm{sin}}$ and $f_{\mathrm{cos}}$ for the three waveforms of Fig. 3. The expression of $f_{\mathrm{cos}}$ in the case of pulse-actuation uses the assumption that $\tau<<1$, as in [22], i.e. that the pulses have a short duration with respect to the period of the oscillation.

It is remarkable that $f_{D C}, f_{\text {sin }}$ and $f_{\text {cos }}$ are independent of $\omega$, except $f_{\text {cos }}$ in the case of pulse actuation. The steady-state regime is determined by solving the nonlinear system of equations:

$$
\begin{aligned}
& B\left(A_{0}, R\right) \stackrel{\Delta}{=} \delta f_{D C}-A_{0}=0, \\
& S\left(A_{0}, R, \omega\right) \stackrel{\Delta}{=} \delta \frac{f_{\text {sin }}}{R\left(1-A_{0}\right)}-\left(1-\omega^{2}\right)=0,(7-\mathrm{b}) \\
& C\left(A_{0}, R, \omega\right) \stackrel{\Delta}{=} 2 \delta v_{0} \frac{f_{\text {cos }}}{R\left(1-A_{0}\right)}-\frac{\omega}{Q}=0, \quad(7-\mathrm{c})
\end{aligned}
$$

corresponding to the averaged dynamics of (3), as derived in Appendix A. This system may have several solutions, the stability of which is studied in section 4 . Note that (7-a) defines an implicit relation between $A_{0}$ and $R$ (or equivalently between $A_{0}$ and $A$ ). The fact that these two quantities are cross-dependent shows that it is not possible to determine the static component of the displacement $A_{0}$ through a static analysis of (3) alone.

\section{Practical determination of steady-state response curves}

The solutions of (7) have no closed-form expressions in terms of the parameters $\delta, v_{0}$ and $Q$, so they must be worked out with a numerical root-finding procedure (e.g. Newton-Raphson). Alternatively, one may proceed as follows: 
1. Assume $Q$ is known.

2. Choose a value of $R$ between 0 and 1 and $\delta$ between 0 and $\delta_{s p i}$.

3. Solve (7-a) for $A_{0}$. Pick the solution (if any) between 0 and $a_{s p i}$.

4. Solve (7-b) for $\omega$.

5. Solve (7-c) for $v_{0}$.

The only complex step in the procedure is step (3), which requires solving a polynomial of the third degree (this can be performed numerically or analytically), while steps (4) and (5) are immediate. If the polynomial of step (3) has no root between 0 and $a_{s p i}$, one should set $v_{0}$ to "not a number", meaning that no oscillation is possible for the chosen values of $Q, R$ and $\delta$. After repeating this process for different values of $R$ and $\delta$, one may then plot the color maps or contour lines of $v_{0}(R, \delta), v_{0}(\omega, \delta)$ or $v_{0}\left(A_{0}, \delta\right)$.

As we have just shown, solving for the steady-state is rather complex. However, the resonant pull-in condition can be established with more ease, as illustrated in the following sub-section.

\section{Determination of the resonant pull-in condition}

1 Stability of the steady-state regime

Let us denote by $\left(A_{0}^{*}, R^{*}, \omega^{*}\right)$ a solution of (7). A necessary and sufficient condition for this equilibrium to be stable is that the Jacobian of (A-14):

$\mathbf{J}=\left(\begin{array}{ccc}-\frac{1}{Q} & \frac{\partial S}{\partial \omega} & \frac{D S}{D R} \\ -2 \omega & \frac{\partial C}{\partial \omega} & \frac{D C}{D R} \\ R & 0 & 0\end{array}\right)$

verifies the Routh-Hurwitz criterion, where the derivative operator

$\frac{D}{D R}=\frac{\partial}{\partial R}+\frac{\partial}{\partial A_{0}} \frac{\partial A_{0}}{\partial R}$

accounts for the cross-dependence of $A_{0}$ and $R$. In (9),

$\frac{\partial A_{0}}{\partial R}=-\frac{\partial B}{\partial R} / \frac{\partial B}{\partial A_{0}}$

is obtained by applying the implicit function theorem to (7-a). The Routh-Hurwitz criterion consists in a set of inequalities which must be verified by the coefficients of the characteristic polynomial of $\mathbf{J}$, $P(\lambda)=\operatorname{det}\left(\mathbf{J}-\lambda \mathbf{I}_{3}\right)$, for the system to be stable:

$\frac{1}{Q}-\frac{\partial C}{\partial \omega}>0, \quad 2 \omega \frac{\partial S}{\partial \omega}-R \frac{D S}{D R}-\frac{1}{Q} \frac{\partial C}{\partial \omega}>0, \quad \frac{D C}{D R} \frac{\partial S}{\partial \omega}-\frac{D S}{D R} \frac{\partial C}{\partial \omega}>0$.

One may verify that, for all waveforms, the first inequality is always met provided $Q>0$. Furthermore, the second inequality is also met provided $A_{0}^{*}<a_{s p i}$. Since these two conditions are always met in practice, the stability of the oscillation boils down to: 
$\frac{D C}{D R} \frac{\partial S}{\partial \omega}-\frac{D S}{D R} \frac{\partial C}{\partial \omega}>0$

This condition can be used to complement the numerical procedure described in section 3-2 and discriminate stable solutions of (7) from unstable ones. However, there is not much physical insight gained from numerically evaluating this quantity, so it is interesting to go one step further and try to derive a resonant pull-in condition from (12).

\section{Resonant pull-in condition}

By definition, the system is at the resonant pull-in condition when in a state $\left(A_{0}^{r p i}, R^{r p i}, \omega^{r p i}\right)$ which verifies (7) and

$$
\frac{D S}{D R} \frac{\partial C}{\partial \omega}-\frac{D C}{D R} \frac{\partial S}{\partial \omega}=0
$$

The resonant pull-in condition is defined by a set of four equations, (7) and (13), with three parameters $\left(\delta, Q\right.$ and $\left.v_{0}\right)$ and three unknowns $\left(A_{0}^{r p i}, R^{r p i}\right.$ and $\left.\omega^{r p i}\right)$. It is then a tedious but straightforward process of algebraic manipulation to remove four of these quantities and obtain a single relation between the two remaining ones. The simplest way to do that, which also leads to the most meaningful relations, is to eliminate $\delta, Q, v_{0}$ and $\omega^{r p i}$ from (7) and (13) and derive a waveform-dependent relation of the form $R^{r p i}=f_{r p i}\left(A_{0}^{r p i}\right)$. This relation defines an "upper bound" for the solutions of (7), in the sense that $\left(A_{0}^{*}, R^{*}, \omega^{*}\right)$ is stable provided $R^{*}<f_{r p i}\left(A_{0}^{*}\right)$ (and unstable otherwise).

In the case of square-wave actuation, it is found that:

$$
R^{r p i}=\frac{1-3 A_{0}^{r p i}}{\sqrt{3-6 A_{0}^{r p i}}}
$$

in the case of sine-wave actuation:

$$
R^{r p i}=\left(1-3 A_{0}^{r p i}\right) \sqrt{\frac{6}{11-18 A_{0}^{r p i}-9 A_{0}^{r p i 2}+\sqrt{13+36 A_{0}^{r p i}-306 A_{0}^{r p i 2}+324 A_{0}^{r p i 3}+81 A_{0}^{r p i 4}}}},
$$

and, in the case of pulse-actuation ${ }^{2}$ :

$R^{r p i}=\sqrt{\frac{1-3 A_{0}^{r p i}}{1+6 A_{0}^{r p i}}}$.

Note that these relations not only predict the occurrence of resonant pull-in but also that static pull-in takes place when $A_{0}^{r p i}=a_{s p i}=1 / 3$, since in that case we find $R^{r p i}=0$, independently of the waveform. On the other hand, the maximal oscillation amplitude is waveform-dependent: it is obtained when $A_{0}^{r p i}=0$ (a situation which may happen when a very small electrostatic bias is used, as in [18]). In that case, we find for square-wave actuation:

\footnotetext{
${ }^{1}$ This inequality is derived in [11] using a more "physical" approach, in the case when $A_{0}=0$. An alternative approach to the determination of this inequality is given in appendix A.

${ }^{2}$ In that case, we find that $\frac{\partial C}{\partial \omega}=\frac{C}{\omega}=0$, and the pull-in condition (13) simplifies to: $\frac{D C}{D R}=0$.
} 
$R^{r p i}=\frac{1}{\sqrt{3}} \approx 0.58$

which is a well-known result [18]. For sine-wave actuation, the maximal oscillation amplitude is found to be:

$R^{r p i}=\sqrt{\frac{6}{11+\sqrt{13}}} \approx 0.64$

Finally, we find, for pulse-actuation:

$R^{r p i}=1$,

i.e. that a full-gap travel range is possible if the electrostatic bias is very small. It is interesting to note that these values are the same as those obtained in the case of double-sided actuation [20].

One can calculate $\omega^{r p i}$ by combining (7-a) and (7-b) which yields:

$\omega^{*}=\sqrt{\frac{1-3 A_{0}^{*}}{1-A_{0}^{*}}}$,

or equivalently

$A_{0}^{*}=\frac{1-\omega^{* 2}}{3-\omega^{* 2}}$,

which is valid for all waveforms.

It is interesting to note that (14) and (7-a) imply that $A_{0}^{r p i}$ and $R^{r p i}$ (and, consequently, $\omega^{r p i}$ ) depend only on $\delta$ and not on $Q$ or $v_{0}$. There are no closed-form expressions for $A_{0}^{r p i}(\delta), R^{r p i}(\delta)$ or $\omega^{r p i}(\delta)$, but these quantities are plotted in Fig. 4 for the three considered waveforms. Equation (7-c) can then be used to determine, for a given quality factor and a given waveform, the maximal value of $v_{0}$ that can be used without incurring resonant pull-in (i.e. a "voltage pull-in condition", as defined in [19]), as plotted in Fig. 5. 

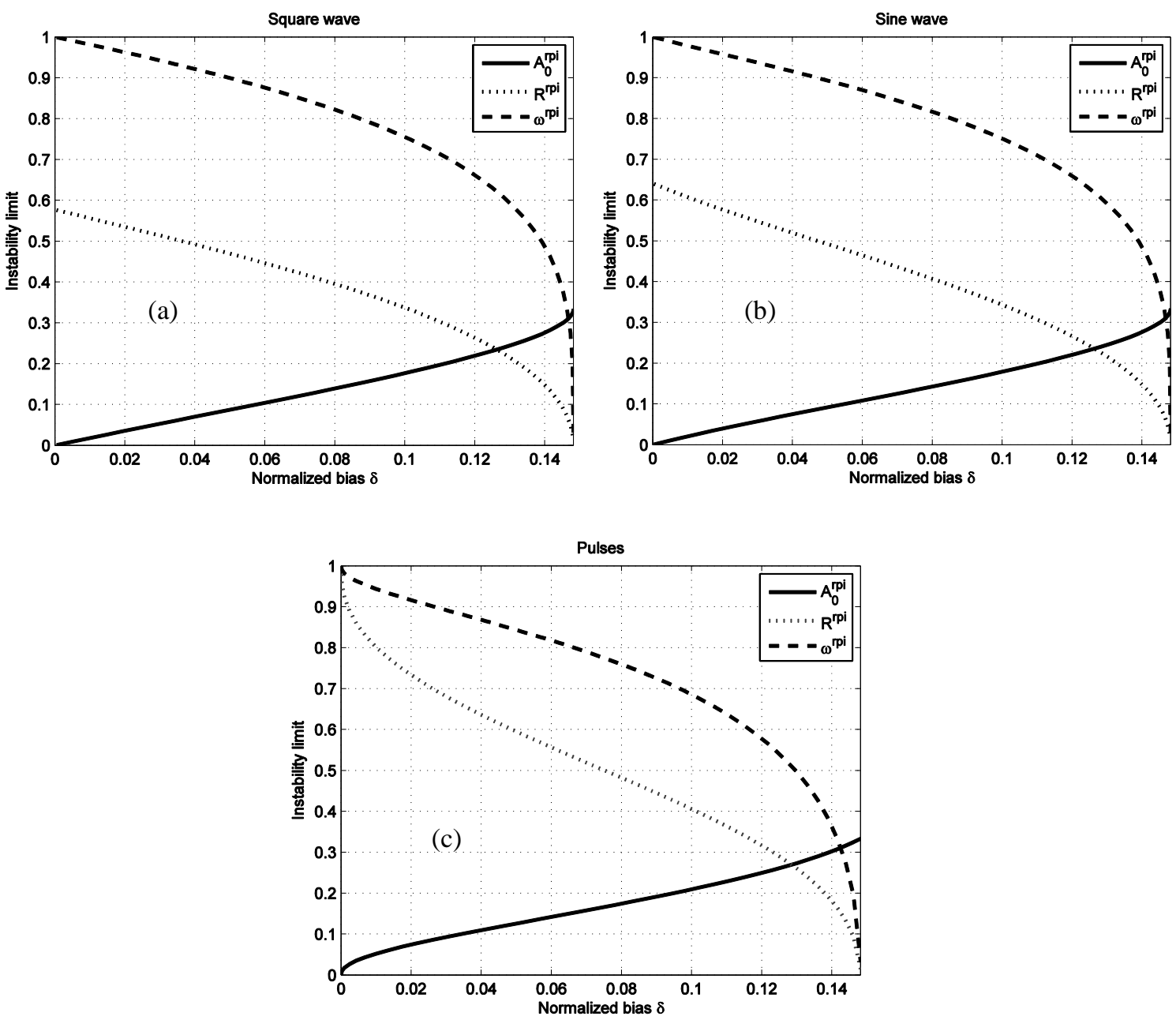

Fig. $4-A_{0}^{r p i}(\delta), R^{r p i}(\delta)$ and $\omega^{r p i}(\delta)$ for the square $(a)$, sine $(b)$ and pulse (c) waveforms, as calculated by (14) and (7-a). 


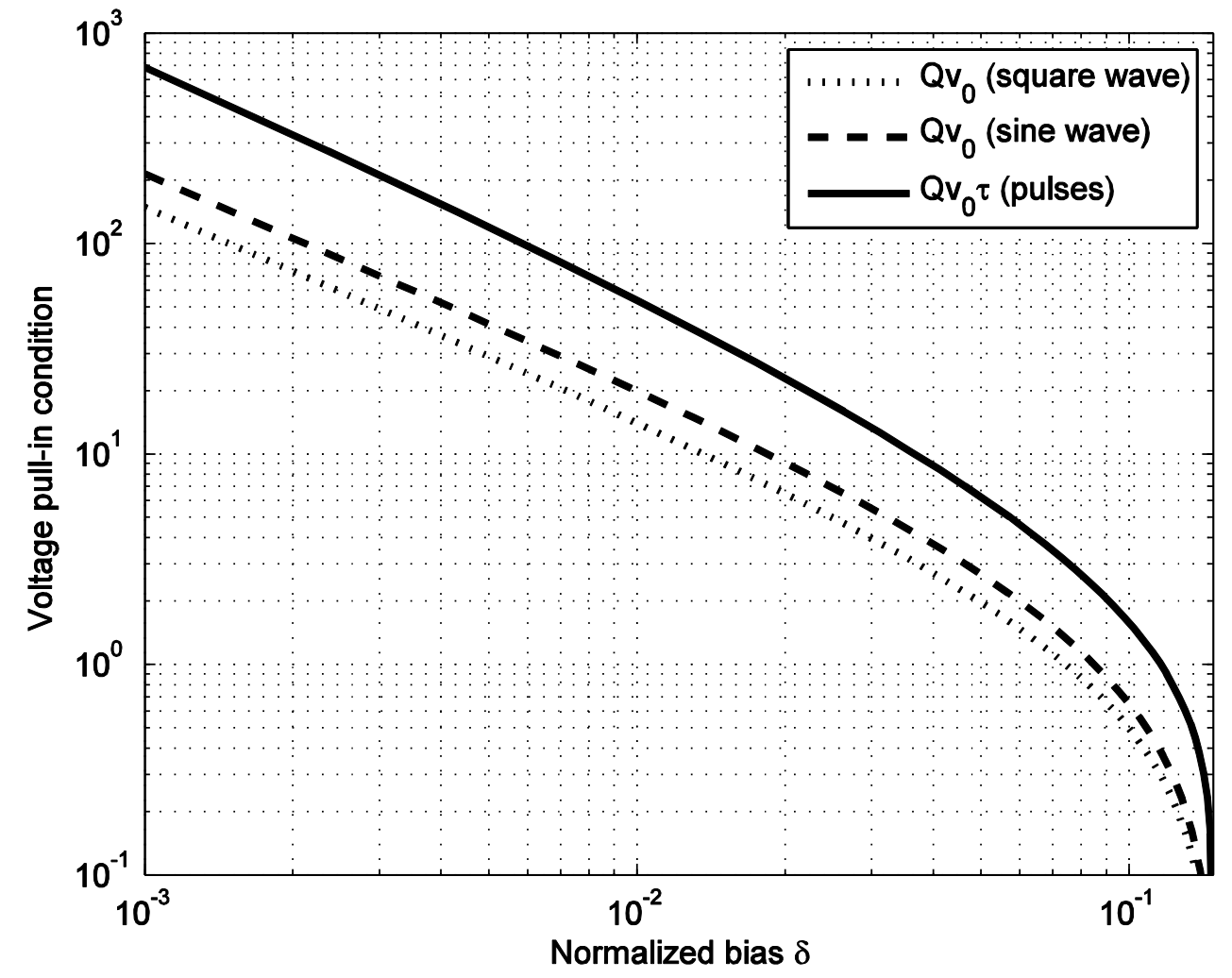

Fig. 5 - Voltage pull-in condition. Note that the plot represents $Q v_{0}(\delta)$ for square and sine wave actuation and $Q v_{0} \tau(\delta)$ for pulse actuation.

These results are compared to those from [19] in the following section.

\section{Application to square-wave actuation}

In this sub-section, we focus on the case of square wave-actuation. Equation (7-c) can then be rewritten as:

$\delta v_{0}=\frac{\pi}{8} \frac{1}{Q} \omega^{r p i}\left(1-A_{0}^{r p i}\right)^{3}\left(1-R^{r p i 2}\right) R^{r p i} .(17)$

Equation (17) should be compared to the resonant pull-in condition found in [19], which can be written, using our notations:

$$
\delta v_{0}=\frac{\pi}{12 \sqrt{3}} \frac{1}{Q} \tilde{\omega}^{r p i}\left(1-\tilde{A}_{0}^{r p i}\right)^{3}
$$

where, for a given DC bias $\delta$, the authors propose to estimate $\tilde{A}_{0}^{r p i}$ in (18) thanks to a static analysis of (1) and to use the following approximation for $\tilde{\omega}^{r p i}$ :

$\tilde{\omega}^{r p i} \approx \sqrt{1-\frac{2 \delta}{\left(1-\tilde{A}_{0}^{r p i}\right)^{3}}}$,

based on a first-order linearization of (1) close to the static solution. 
Transient simulations of (1) are performed with Matlab/Simulink, for different values of $\delta$ and $Q=10$. For each value of $\delta$, the value of $v_{0}$ is increased step by step until resonant pull-in is reached. The smallest value of $v_{0}$ for which the system is unstable is noted down. These results are compared with those obtained with (17) and (18) (Fig. 6). As could be expected, (17) and (18) yield similar results when the DC bias is small $(\delta<<1)$ but overestimate the value of $v_{0}$. For finite values of $\delta$, the two methods no longer agree: while transient simulation of (1) show that (18) fails to predict the correct resonant pull-in condition for finite values of $\delta$, the results obtained with (17) are very accurate.

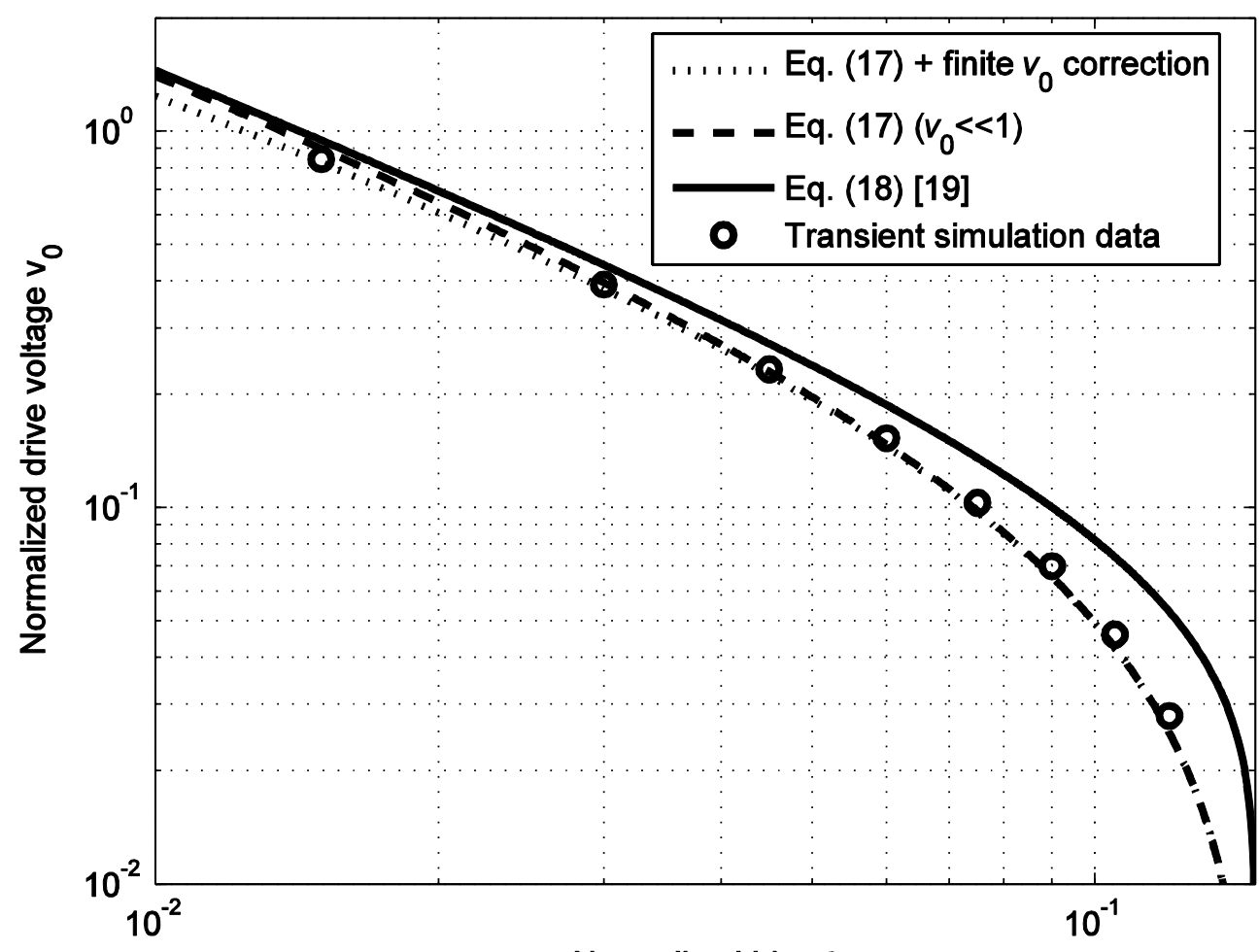

Normalized bias $\delta$

Fig. 6 - Voltage pull-in condition obtained with three different methods compared to transient simulation results. The dashed curve corresponds to the dotted curve of Fig. 5, translated by one decade (since $Q=10$ ).

This discrepancy for finite values of $\delta$ can be attributed to an error made in [19] in deriving (18): it is assumed in [19] that equation (29), reproduced hereafter with our notations:

$A^{3}-A\left(1-A_{0}\right)^{2}+\frac{8 Q \delta v_{0}}{\pi \omega}=0$

is a polynomial of the third degree in $A$ and that resonant pull-in is reached when its discriminant is zero. However, as explained in section $3, A_{0}$ and $A$ are cross-dependent so that (i) one may not derive values of $A_{0}$ and $\omega$ from a static analysis alone and (ii) one cannot consider (20) as a thirddegree polynomial.

In the case of small $\delta$, the difference between the results obtained with both methods and transient simulations can be explained by the fact that the predicted values of $v_{0}$ are then finite and, consequently, fall outside the scope of both studies. However, in the square-wave case, $v^{2}=v_{0}{ }^{2}$ is a constant, and the analysis made in the previous sections still holds, provided one multiplies $f_{D C}$ and 
$f_{\sin }$ by $1+v_{0}^{2}$ in (7). Equations (14-a) and (16) remain valid in spite of this modification. Thus, in order to find the maximal value of $v_{0}$ that can be used without incurring resonant pull-in, one should (i) choose a value of $R^{r p i}$ between 0 and $1 / \sqrt{3}$, (ii) deduce the corresponding $A_{0}^{r p i}$ and $\omega^{r p i}$ with (14a) and (16-a), (iii) eliminate $v_{0}$ from (7-a) and (7-c) and solve the resulting second-degree polynomial for $\delta$, (iv) calculate $v_{0}$ from (7-c). The results obtained with this approach are also represented in Fig. 6 and show an excellent agreement with transient simulation results.

\section{Extension to more complex cases and limits of the study}

\section{Extension to other resonator geometries}

Electrostatically-actuated cantilever beam and clamped-clamped beam resonators with rectangular cross-sections are commonplace in MEMS and NEMS applications. Calculating the resonant pull-in condition for these geometries is more arduous than for parallel-plate resonators. Still this can be achieved by numerically approximating the projection of the electrostatic force on the shape function used to describe the beam deformation in a proper way, and, consequently, the functions $f_{\text {sin }}, f_{\text {cos }}$ and $f_{D C}$.

For example, we start with the Euler-Bernoulli equation, governing the displacement $w$ of an electrostatically-actuated rectangular beam with length $L$, thickness $h$, width $b$, Young's modulus $E$ , viscous damping coefficient $\mu$ and density $\rho$ :

$$
\frac{E b h^{3}}{12} \frac{\partial^{4} w}{\partial x^{4}}+\mu \frac{\partial w}{\partial t}+\rho b h \frac{\partial^{2} w}{\partial t^{2}}=\frac{\varepsilon_{0} b}{2} \frac{V^{2}}{\left(G_{0}-w\right)^{2}} \chi_{[\alpha L, \beta L]},
$$

where it is supposed that the actuation electrode extends from $x=\alpha L \geq 0$ to $x=\beta L \leq L$, and $\chi_{[\alpha L, \beta L]}$ is the indicator function of $[\alpha L, \beta L]$. Note also that the electrode/beam length is supposedly large with respect to the gap and to the width of the beam, so that the plane capacitor approximation remains valid in a cross-section of the beam. Setting $x=L \tilde{x}, w=G_{0} \tilde{w}$ and $\tilde{t}=\omega_{0} t$, and remembering that $v \ll 1$, (21) becomes:

$$
\frac{E b h^{3}}{12 L^{4}} \frac{\partial^{4} \tilde{w}}{\partial \tilde{x}^{4}}+\mu \omega_{0} \frac{\partial \tilde{w}}{\partial \tilde{t}}+\rho b h \omega_{0}^{2} \frac{\partial^{2} \tilde{w}}{\partial \tilde{t}^{2}}=\frac{\varepsilon_{0} b V_{b}^{2}}{2 G_{0}^{3}} \frac{1+2 v}{(1-\tilde{w})^{2}} \chi_{[\alpha L, \beta L]} .
$$

Letting $\tilde{w}(\tilde{x}, \tilde{t})=a(\tilde{t}) w_{1}(\tilde{x})$, where $w_{1}(\tilde{x})$ is the first beam eigenmode, normalized so that $\max \left(w_{1}\right)=1$, a Galerkin procedure is applied to (22). This leads to the projected non-dimensional dynamics:

$$
\ddot{a}+\frac{\dot{a}}{Q}+a=\delta(1+2 v) \int_{\alpha}^{\beta} \frac{w_{1}(\tilde{x})}{\left(1-a w_{1}(\tilde{x})\right)^{2}} d \tilde{x},
$$

where

$$
\delta=\frac{1}{\Gamma_{1}} \frac{\varepsilon_{0}}{E} \frac{L^{4} V_{b}^{2}}{h^{3} G_{0}^{3}}, Q=\Gamma_{2} \frac{\sqrt{E \rho}}{\mu} \frac{b h^{2}}{L^{2}}
$$

with $\Gamma_{1} \approx 33.12$ and $\Gamma_{2} \approx 6.46$ for a clamped-clamped beam, and $\Gamma_{1} \approx 0.515$ and $\Gamma_{2} \approx 1.015$ for a cantilever beam. 
Approximate expressions for the right-hand side of (23) can be obtained as explained in appendix B, guaranteeing that the relative error between the projected force and its approximation is bounded for $a<1$. For example, in the trivial case when $\alpha=0$ and $\beta=1$, the projected dynamics become:

$\ddot{a}+\frac{\dot{a}}{Q}+a \approx 0.523 \delta(1+2 v) \frac{1+0.017 a}{(1-a)^{3 / 2}}$,

in the case of a clamped-clamped beam, and

$$
\ddot{a}+\frac{\dot{a}}{Q}+a \approx 0.392 \delta(1+2 v)\left(\frac{1+0.783 a}{1-a}+(0.531+0.114 a) \log (1-a)\right)
$$

for a cantilever beam. The resulting reduced-order models can then be averaged over one period to yield the equivalent of system (7) for a deformable beam. The expressions of $f_{D C}, f_{\text {sin }}$ and $f_{\text {cos }}$ can be derived as shown in appendix $\mathrm{C}$ of this paper (the calculation of $f_{D C}$ is illustrated in appendix D). The same process as in section 4 can then be applied to determine resonant pull-in conditions: these are represented in Fig. 7 and Fig. 8, in the case when $\alpha=0$ and $\beta=1$. Note that this last step must be performed numerically: while it is simple to use (13) and the steady-state equations to obtain an implicit relation between $A_{0}^{r p i}$ and $R^{r p i}$ where only these two quantities appear, it is (to the author's knowledge) impossible to make it explicit, as opposed to the parallel-plate case. Finally, in the more general case when the lengths of the beam and the actuating electrode are different, one should replace $N(a)$ in (6) by a parameterized reduced-order model $\hat{N}(a, \boldsymbol{\theta})$, established as in appendix B, and follow the same procedure as above. 

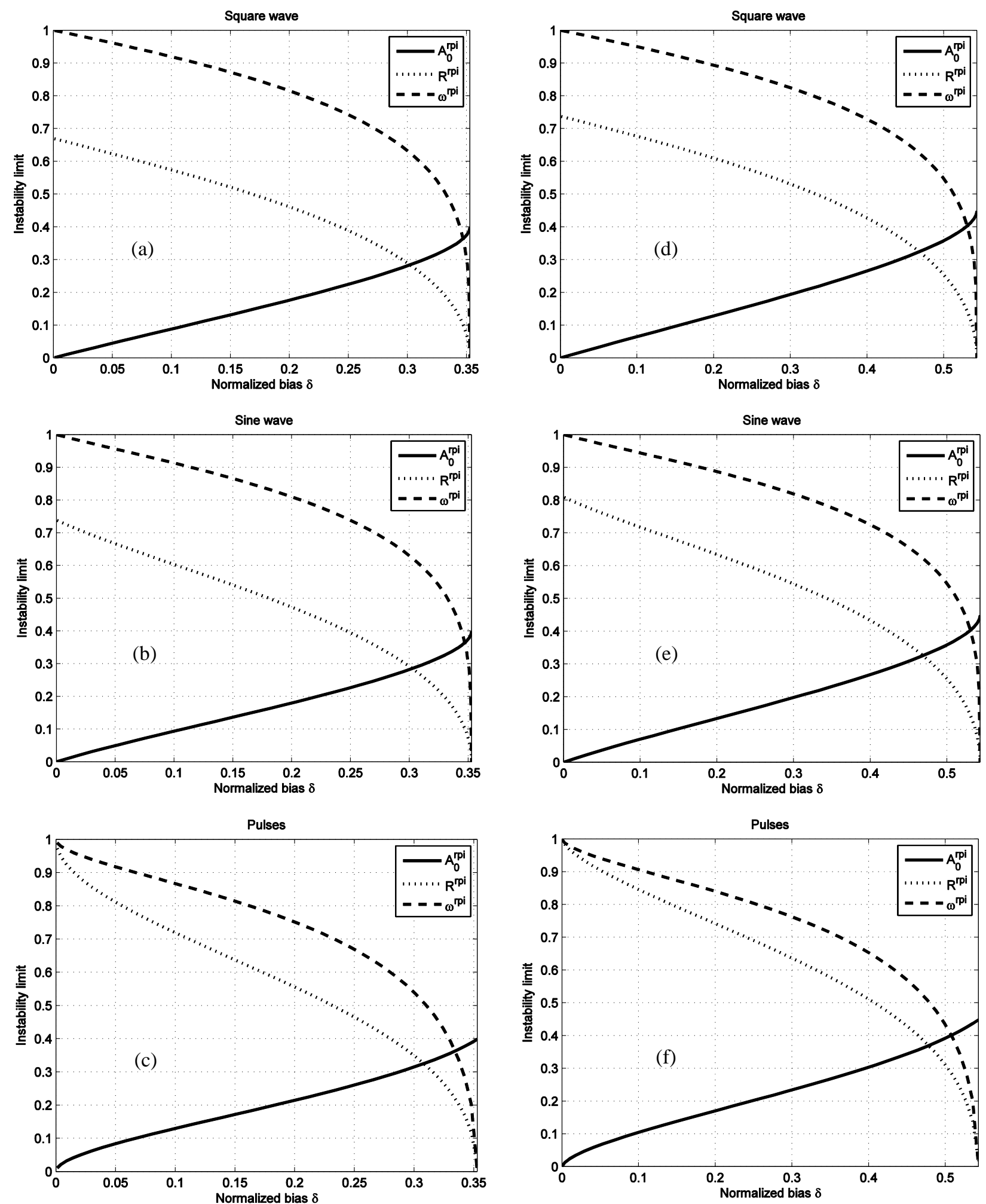

Fig. 7 - $A_{0}^{r p i}(\delta), R^{r p i}(\delta)$ and $\omega^{r p i}(\delta)$ for the three waveforms, in the case of a clamped-clamped beam $(a, b, c)$ and in the case of a cantilever beam $(d, e, f)$. 
(a)

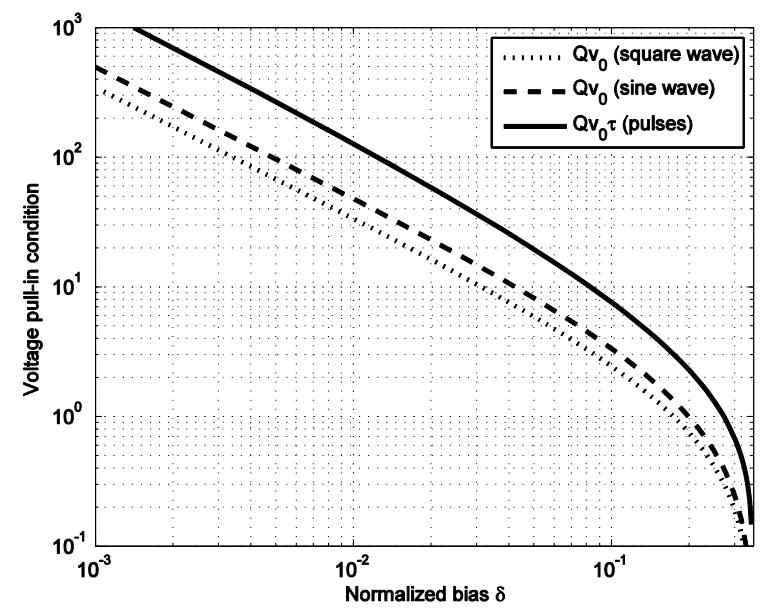

(b)

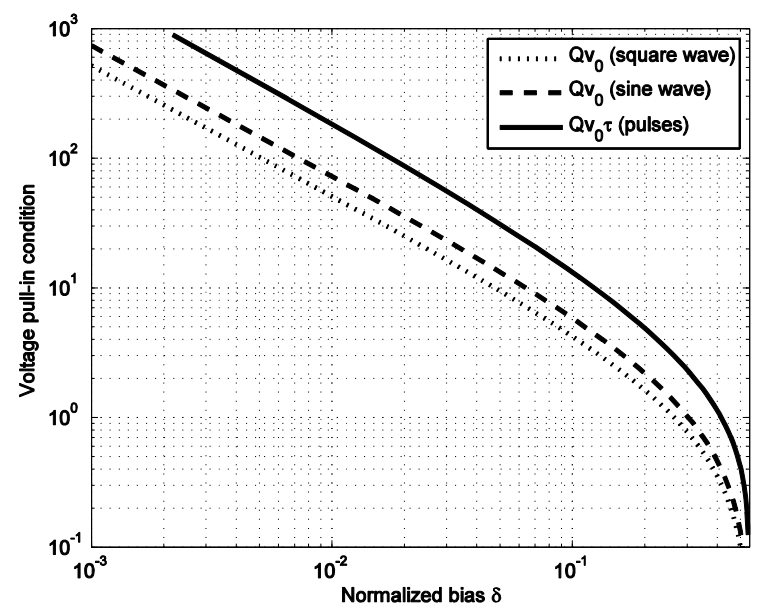

Fig. 8 -Voltage pull-in condition for a clamped-clamped beam (a) and for a cantilever beam (b).

\section{Extension and limits of the study}

This study is admittedly limited to an idealized framework and the presented results should always be quantitatively questioned when working in an experimental context. In this section, the main limitations of our study are listed, in order of ascending difficulty, and some guidelines for tackling them are given.

a) Non-ideality of the geometry

First of all, it must be stressed that the reduced-order models proposed in section 5.1 are valid as long as the plane capacitor approximation holds in a cross-section of the beam. However, this hypothesis is not always verified, in particular in nanoscale devices. Geometries with small length/gap or width/gap ratios require either that fringing fields be accounted for in the expression of the electrostatic force (this may also be done with the approach proposed in [23]), or that other reduced-order modeling strategies be used, such as the fully numerical approach proposed in [25]. Regardless of which approach is used, the resonant pull-in condition may still be derived from (13), provided the dynamics of the device can be captured with only one mode. In spite of the added complexity of deriving a model of the electrostatic force, no new parameter is introduced in the equations and the resonant pullin condition may still be obtained at no great cost.

Other interesting "non-ideal" geometries are asymmetric double-sided resonators, to which the methods presented in this paper can readily be applied.

b) Presence of other nonlinearities

When other nonlinearities (e.g. caused by beam elongation, squeezed-film damping, etc.) are present, the set of four equations defining the resonant pull-in condition contains more than four parameters. Consequently, it is not possible, in such a case, to derive "nice" closed-form expressions of the resonant pull-in condition, such as (14). Yet, it is still possible to use (13) to obtain resonant-pull-in curves parameterized by the supplementary parameters.

For example, beam elongation is equivalent to Duffing stiffening, with a geometry-dependent Duffing coefficient $\gamma$. If this phenomenon is taken into account, the resonant pull-in condition, can be worked out as previously, by eliminating $\delta, Q, v_{0}$ and $\omega^{r p i}$ from (13) and (7). The resulting relation between $A_{0}^{r p i}, R^{r p i}$ is then parameterized by $\gamma$ (the case $\gamma=0$ corresponding to Fig. 7 and 8). 
c) Non-ideality of the feedback loop

In the present paper, it is assumed that the displacement of the structure $(a)$ or its velocity $(\dot{a})$ is directly measured and the waveform used for actuation is perfectly in quadrature with $a$. This makes it possible to "nicely" separate the influence of the actuation voltage from that of the bias voltage in the steady-state equations, and yields closed-form expressions of the resonant pull-in condition ${ }^{3}$. However a phase delay $\varphi$ may be present in the feedback loop, which also introduces a supplementary parameter in (7) and (13) and should be treated as in 5.2.b.

For example, detection nonlinearities may make it practically difficult to generate the actuation waveform in quadrature with the displacement. A typical example is when a capacitive detection scheme is used: first of all, the detected signal is polluted by "feedthrough", i.e. the detected signal is the superposition of the motional signal $a$ and of the actuation waveform, which impacts the loop delay. Also, the detected signal becomes heavily distorted as a increases (the capacitance being a nonlinear function of $a$ ), which may make it difficult to ensure quadrature in the loop, unless some particular electronics are used (e.g. a PLL).

Thus, one should use the present study "as is" only when the feedback loop correctly enforces quadrature over the whole range of possible values of $a$. Otherwise, one should adapt the theory to the non-ideal framework.

d) Interaction with other modes

It should be pointed out that the present study is limited to the case when the resonator can be modeled as a system with a single degree-of-freedom. It should be used with caution for complex structures because of possible modal interactions induced by electrostatic coupling. In particular, as $a$ increases and becomes finite, it may be incorrect to neglect the components of the electrostatic force at $2 \omega, 3 \omega$, etc. and their effect on the dynamics of the system [26].

\section{Conclusion}

In this paper, a method for determining the steady-state dynamics and the resonant pull-in condition of MEMS-based oscillators actuated from only one side was proposed. After illustrating this method in the case of a parallel-plate resonator, this approach was extended to clamped-clamped beam and cantilever beam resonators. The proposed approach is original as it accurately captures the crossdependence of the static and dynamic components of the resonator displacement. This is in contrast with the double-sided case (in which the displacement has no static component) [20].

The proposed approach also predicts pull-in for $0<\delta \leq \delta_{s p i}$, i.e. from "purely resonant" to static pullin, whereas previous studies of closed-loop single-sided actuation are either limited to the case when the bias voltage is very small with respect to the static pull-in voltage (i.e. $\delta \ll \delta_{s p i}$ ) and square-wave actuation [18] or fail to accurately predict pull-in [19], as comparison with the proposed method and transient simulations has shown. The increased displacement range obtained when using pulsed actuation compared to sine-wave or square-wave actuation is also an interesting outcome of this study.

In the case of flexible beam resonators, the proposed approach is used in conjunction with a singlemode approximation of the nonlinear actuation forces, which is valid across the whole gap [23], and resonant pull-in conditions are also established. It should be noted that the calculations resulting from this approach are simpler than those which would be obtained with the reduced-order modeling approach proposed in [6], which requires several modes to capture the large-amplitude behavior of

\footnotetext{
${ }^{3}$ At least for parallel-plate resonators. In the other geometries, the pull-in condition can easily be established using a numerical procedure, as shown in section 5.1 and 5.2.a.
} 
electrostatically-actuated MEMS beams ${ }^{4}$. Moreover, the reduced-order modeling approach proposed in [6] is inaccurate when used with only one mode (dramatically so if used in conjunction with oneharmonic harmonic balance analysis, as shown in [27]). Because of this added complexity, and also because the open-loop problem is more involved than the closed-loop problem, no resonant pull-in conditions are established in [7-8].

Consequently, extending the method of the present paper to the open-loop case, in which the frequency is set and the phase varies, is the subject of ongoing work. Future work will also be devoted to accounting for non-idealities described in section 5-2, in particular to feedback loop non-idealities such as phase-delay and capacitive feedthrough.

\section{Appendix A - Averaged dynamics of the system}

Let

$a(t)=A_{0}+A(t) \sin \psi(t)$,

where $A(t)$ and $\psi(t)$ are assumed to be slowly-varying functions of time. Following [11], we define

$\sigma=\frac{\dot{A}}{A}$

the rate of change of the amplitude and

$\omega=\dot{\psi} .(\mathrm{A}-3)$

Differentiating (A-1) yields:

$\dot{a}=A(\sigma \sin \psi+\omega \cos \psi), \quad(\mathrm{A}-4)$

$\ddot{a}=A\left(\left(\sigma^{2}+\dot{\sigma}-\omega^{2}\right) \sin \psi+(2 \sigma \omega+\dot{\omega}) \cos \psi\right)$.

Injecting (A-1), (A-4) and (A-5) in (3) and taking the average, the projection on $\sin \psi$ and the projection on $\cos \psi$ over one period of the resulting equations leads to:

$A_{0}=\delta f_{D C}, \quad(\mathrm{~A}-6)$

$\dot{\sigma}=\delta \frac{f_{\text {sin }}}{A}-\left(1-\omega^{2}\right)-\frac{\sigma}{Q}-\sigma^{2}$

$\dot{\omega}=2 \delta v_{0} \frac{f_{\mathrm{cos}}}{A}-\frac{\omega}{Q}-2 \sigma \omega$

Equations (A-2), (A-7) and (A-8) are a nonlinear autonomous dynamical system. The equilibrium is reached when the time-derivatives equal zero, i.e.

$\dot{A}=0 \Leftrightarrow \sigma=0$,

(A-9)

\footnotetext{
${ }^{4}$ As stated by the authors of [6-7] themselves: "A minimum of three modes were employed in the reduced-order model used to predict the transient and steady-state responses reported here. This corresponds to a sixdimensional phase space. As a result, generating the device stability maps is a nontrivial task."
} 
$\delta \frac{f_{\text {sin }}}{A}-\left(1-\omega^{2}\right)=0$

$\delta \frac{f_{\mathrm{cos}}}{A}-\frac{\omega}{Q}=0$.

One may introduce the reduced amplitude $R$ defined by

$R=\frac{A}{1-A_{0}}$

in all of these equations, since

$\sigma=\frac{\dot{A}}{A}=\frac{\left(1-A_{0}\right) \dot{R}}{\left(1-A_{0}\right) R}=\frac{\dot{R}}{R}$.

Using this notation, the dynamical system can be written, with the notations defined in section 3 , as:

$\left\{\begin{array}{l}\dot{\sigma}=S\left(A_{0}, R, \omega\right)-\frac{\sigma}{Q}-\sigma^{2} \\ \dot{\omega}=C\left(A_{0}, R, \omega\right)-2 \sigma \omega \\ \dot{R}=\sigma R\end{array}\right.$.

The stability of the system is studied in section 5, by applying the Routh-Hurwitz criterion to the jacobian matrix of (A-14).

Alternatively, one may derive a stability condition by assuming that $\dot{a}$ is given by:

$\dot{a}=A \omega \cos \psi \triangleq V\left(1-A_{0}\right) \cos \psi(\mathrm{A}-15)$

instead of (A-3), where $V$ would be a reduced velocity. The dynamics of the system are then governed by:

$\dot{V}=2 \delta v_{0} \frac{f_{\cos }}{1-A_{0}}-\frac{V}{Q} \triangleq \mathrm{K}\left(A_{0}, R, V\right)$

$\Sigma\left(A_{0}, R, V\right) \triangleq \delta \frac{f_{\text {sin }}}{R\left(1-A_{0}\right)}-\left(1-\frac{V^{2}}{R^{2}}\right)=0$,

while (A-6) remains unchanged. The stability of this dynamic system is guaranteed provided

$\frac{\Delta \mathrm{K}}{\Delta V} \triangleq \frac{\partial \mathrm{K}}{\partial V}+\frac{\partial \mathrm{K}}{\partial R} \frac{\partial R}{\partial V}+\frac{\partial \mathrm{K}}{\partial A_{0}} \frac{\partial A_{0}}{\partial V}<0$

Taking the total derivative of $\Sigma\left(A_{0}, R, V\right)$ with respect to $V$ yields:

$\frac{\partial R}{\partial V}=-\frac{\partial \Sigma}{\partial V} / \frac{D \Sigma}{D R},(\mathrm{~A}-19)$

where the differential operator $D$ is the same as in section 4 : 


$$
\frac{D}{D R}=\frac{\partial}{\partial R}-\left(\frac{\partial B}{\partial R} / \frac{\partial B}{\partial A_{0}}\right) \frac{\partial}{\partial A_{0}}
$$

Differentiating $B\left(A_{0}, R\right)$ with respect to $V$ yields:

$$
\frac{\partial A_{0}}{\partial V}=\frac{\partial A_{0}}{\partial R} \frac{\partial R}{\partial V}=-\left(\frac{\partial B}{\partial R} / \frac{\partial B}{\partial A_{0}}\right) \frac{\partial R}{\partial V} .
$$

Thus, the stability condition may be expressed as:

$$
\frac{\partial \mathrm{K}}{\partial V}-\left(\frac{D \mathrm{~K}}{D R} / \frac{D \Sigma}{D R}\right) \frac{\partial \Sigma}{\partial V}<0 .
$$

At equilibrium, this equation is actually equivalent to (12), because $C=0$ implies that

$$
\frac{\partial \mathrm{K}}{\partial V}=R \frac{\partial C}{\partial V}
$$

and

$$
\frac{D \mathrm{~K}}{D R}=R \frac{D C}{D R} .
$$

Furthermore, we have:

$$
\Sigma\left(A_{0}, R, V\right)=S\left(A_{0}, R, \frac{V}{R}\right) .
$$

Finally, the jacobian determinant of the transformation from $(R, \omega)$ coordinates to $(R, V)$ coordinates is positive. Thus, (A-22) is equivalent to (12).

\section{Appendix B - Reduced-order modelling of MEMS beams}

By and large, the reduced order models given in the paper are obtained as in [23], by minimizing the relative error between a numerical estimation of the projection of the electrostatic force on the first beam eigenmode, denoted by $w_{1}$ :

$$
N(a)=\int_{\alpha}^{\beta} \frac{w_{1}(\tilde{x})}{\left(1-a w_{1}(\tilde{x})\right)^{2}} d \tilde{x}
$$

and a model parameterized by $\boldsymbol{\theta}, \hat{N}(a, \boldsymbol{\theta})$. The relative error is summed over a set of chosen modal amplitudes $a_{i}$, so that the optimal parameters are given by:

$$
\boldsymbol{\theta}_{\text {opt }}=\arg \min \sum_{i}\left(1-\frac{\hat{N}\left(a_{i}, \boldsymbol{\theta}\right)}{N\left(a_{i}\right)}\right)^{2} .
$$

For a clamped-clamped beam, provided $\beta=1-\alpha$ (the actuation electrode is centered), the model has the following form: 


$$
\hat{N}(a, \theta)=\frac{N_{C C}}{(1-a)^{3 / 2}}(1+\theta a) .
$$

For a cantilever beam, provided $\beta=1$ (the actuation electrode extends to the tip of the beam), it can be chosen as:

$$
\hat{N}\left(a, \theta_{1}, \theta_{2}, \theta_{3}\right)=N_{C A}\left(\frac{1+\theta_{1} a}{1-a}+\left(\theta_{2}+\theta_{3} a\right) \log (1-a)\right) .
$$

The coefficients $N_{c c}$ and $N_{C A}$ are calculated so that $\hat{N}(0, \boldsymbol{\theta})=N(0)$, i.e. the model and the projection of the electrostatic force coincide when $a=0$. The structure of the model, independently of its parameters, guarantees that the asymptotic behavior (as $a$ goes to 1 ) of $\hat{N}(a, \boldsymbol{\theta})$ is the same as that of $N(a)$, as shown in [23], so that the relative error is bounded across the whole gap.

To obtain the parameters appearing in (25) and (26), (B-2) is solved: this is a trivial linear least squares problem. The error is minimized over a set of 100 values $a_{i}$ of the modal amplitude, uniformly spaced between -0.99 and 0.99 .

Introducing the log function in the ROM of the cantilever beam (B-4) gives better results than the approach proposed in [23]. This approach was already used in [20].

Note that the exact values of the model parameters depend not only on the chosen abscissas, but also on the geometry of the actuation electrode. The numerical values given in the paper are only valid when the actuation electrode is the same length as the beam. However, the approach of [23] can also be used to approximate integrals of the form:

$$
N(a, n, p)=\int_{\alpha}^{\beta} \frac{w_{1}^{p}}{\left(1-a w_{1}\right)^{n}} d \tilde{x},
$$

and thus account for fringing field effects $(n=p=1)$ or other nonlinear forces.

\section{Appendix C - Calculations of $f_{D C}, f_{\text {sin }}$ and $f_{\text {cos }}$ for clamped-clamped and cantilever beams}

In order to calculate $f_{D C}, f_{\text {sin }}$ and $f_{\text {cos }}$ in the case of cantilever or clamped-clamped beams, one should replace $N(a)$ in (6) by the reduced-order model $\hat{N}(a, \boldsymbol{\theta})$ obtained in appendix B. The resulting integrals can all be expressed in terms of Gauss hypergeometric function ${ }_{2} F_{1}$ [24].

In particular, the following equality is useful for determining $f_{D C}$ :

$\frac{1}{2 \pi} \int_{0}^{2 \pi} \frac{d t}{(1-R \sin (t))^{n}}={ }_{2} F_{1}\left(\frac{n}{2}, \frac{n+1}{2}, 1, R^{2}\right)$,

which is valid for any real $n$.

We also have:

$$
\frac{1}{2 \pi} \int_{0}^{2 \pi} \log (1-R \sin (t)) d t=\log \left(\frac{1}{2}\left(1+\sqrt{1-R^{2}}\right)\right)
$$


and

$\frac{1}{2 \pi} \int_{0}^{2 \pi}(1-R \sin (t)) \log (1-R \sin (t)) d t=1-\sqrt{1-R^{2}}+\log \left(\frac{1}{2}\left(1+\sqrt{1-R^{2}}\right)\right)$.

$f_{\text {sin }}$ can be determined in the same fashion using:

$\frac{1}{\pi} \int_{0}^{2 \pi} \frac{\sin (t) d t}{(1-R \sin (t))^{n}}=n R_{2} F_{1}\left(\frac{n+1}{2}, \frac{n+2}{2}, 2, R^{2}\right)$

$\frac{1}{\pi} \int_{0}^{2 \pi} \sin (t) \log (1-R \sin (t)) d t=-\frac{2 R}{1+\sqrt{1-R^{2}}}$

and

$\frac{1}{\pi} \int_{0}^{2 \pi} \sin (t)(1-R \sin (t)) \log (1-R \sin (t)) d t=-R\left(\frac{1}{1+\sqrt{1-R^{2}}}+\frac{1}{2}+\log \left(\frac{1}{2}\left(1+\sqrt{1-R^{2}}\right)\right)\right)$.

It is immediate to derive the waveform-dependent function $f_{\text {cos }}$ in the case of pulse actuation. For the square-wave case, the following formulas must be used:

$\frac{1}{\pi} \int_{0}^{2 \pi} \operatorname{sign}(\cos (t)) \frac{\cos (t) d t}{(1-R \sin (t))^{n}}=\frac{2}{(n-1) \pi R}\left(\frac{1}{(1-R)^{n-1}}-\frac{1}{(1+R)^{n-1}}\right), n \neq 1$,

$\frac{1}{\pi} \int_{0}^{2 \pi} \operatorname{sign}(\cos (t)) \frac{\cos (t) d t}{(1-R \sin (t))}=\frac{2}{\pi R} \log \left(\frac{1+R}{1-R}\right)$

$\frac{1}{\pi} \int_{0}^{2 \pi} \cos (t) \operatorname{sign}(\cos (t)) \log (1-R \sin (t)) d t=\frac{2}{\pi R}((1+R) \log (1+R)-(1-R) \log (1-R)-2 R)$,

$\frac{1}{\pi} \int_{0}^{2 \pi} \cos (t) \operatorname{sign}(\cos (t))(1-R \sin (t)) \log (1-R \sin (t)) d t=\frac{1}{\pi R}\left((1+R)^{2} \log (1+R)-(1-R)^{2} \log (1-R)-2 R\right)$ (C-10)

Finally, in the case of sine-wave actuation, we have:

$$
\begin{aligned}
& \frac{1}{\pi} \int_{0}^{2 \pi} \frac{\cos (t)^{2} d t}{(1-R \sin (t))^{n}}={ }_{2} F_{1}\left(\frac{n}{2}, \frac{n+1}{2}, 2, R^{2}\right), \quad(\mathrm{C}-11) \\
& \frac{1}{\pi} \int_{0}^{2 \pi} \cos (t)^{2} \log (1-R \sin (t)) d t=\frac{1}{2} \frac{1-\sqrt{1-R^{2}}}{1+\sqrt{1-R^{2}}}+\log \left(\frac{1}{2}\left(1+\sqrt{1-R^{2}}\right)\right),
\end{aligned}
$$

$\frac{1}{\pi} \int_{0}^{2 \pi} \cos (t)^{2}(1-R \sin (t)) \log (1-R \sin (t)) d t=\frac{1}{3}\left(\frac{1}{1+\sqrt{1-R^{2}}}-2 \sqrt{1-R^{2}}\right)+\frac{1}{2}+\log \left(\frac{1}{2}\left(1+\sqrt{1-R^{2}}\right)\right)$, (C-13). 
Note that (C-1), (C-4) and (C-11) can be simplified when $n$ is an integer, as in the parallel-plate case and in the cantilever case, or a half-integer, as in the clamped-clamped case. The corresponding expressions, tabulated from [24], are given in Table C-1.

\begin{tabular}{|c|c|c|c|}
\hline & ${ }_{2} F_{1}\left(\frac{n}{2}, \frac{n+1}{2}, 1, R^{2}\right)$ & ${ }_{2} F_{1}\left(\frac{n+1}{2}, \frac{n+2}{2}, 2, R^{2}\right)$ & ${ }_{2} F_{1}\left(\frac{n}{2}, \frac{n+1}{2}, 2, R^{2}\right)$ \\
\hline$n=\frac{1}{2}$ & $\frac{2}{\pi} \frac{1}{\sqrt{1+R}} \mathrm{~K}$ & $\frac{8}{\pi R^{2}} \frac{1}{\sqrt{1+R}}(\mathrm{~K}-(1+R) \mathrm{E})$ & $\frac{8}{3 \pi R^{2}} \sqrt{1+R}(\mathrm{E}-(1-R) \mathrm{K})$ \\
\hline$n=1$ & $\frac{1}{\sqrt{1-R^{2}}}$ & $\frac{2}{\left(1+\sqrt{1-R^{2}}\right) \sqrt{1-R^{2}}}$ & $\frac{2}{1+\sqrt{1-R^{2}}}$ \\
\hline$n=\frac{3}{2}$ & $\frac{2}{\pi} \frac{\sqrt{1+R}}{1-R^{2}} \mathrm{E}$ & $\frac{8}{3 \pi R^{2}} \frac{\sqrt{1+R}}{1-R^{2}}(\mathrm{E}-(1-R) \mathrm{K})$ & $\frac{8}{\pi R^{2}} \frac{1}{\sqrt{1+R}}(\mathrm{~K}-(1+R) \mathrm{E})$ \\
\hline
\end{tabular}

Table C-1 - Expressions of Gauss' hypergeometric function for half-integer values of $n$ (clamped-clamped beams) and for $n=1$ (cantilever beams). In this table, $\mathrm{K}$ (resp. E ) stands for the complete elliptic integral of the first (resp. second) kind, with argument $2 R /(1+R)$.

Appendix D - Example calculation of electrostatic force and $f_{D C}$ for clamped-clamped and cantilever beams

As an example, consider a reduced-order model for a clamped-clamped beam with a centered electrode of arbitrary length:

$\ddot{a}+\frac{\dot{a}}{Q}+a \approx \delta(1+2 v) N_{C C} \frac{1+\theta a}{(1-a)^{3 / 2}}$,

or, equivalently:

$\ddot{a}+\frac{\dot{a}}{Q}+a \approx \delta(1+2 v) N_{C C}\left(\frac{1+\theta}{(1-a)^{3 / 2}}-\frac{\theta}{(1-a)^{1 / 2}}\right)$.

Assuming (A-1) holds, the right-hand side of (D-2) becomes:

$\delta(1+2 v) N_{C C}\left(\frac{1+\theta}{\left(1-\left(A_{0}+A \sin \psi\right)\right)^{3 / 2}}-\frac{\theta}{\left(1-\left(A_{0}+A \sin \psi\right)\right)^{1 / 2}}\right)$.

Using the reduced amplitude (A-12), this becomes:

$\delta(1+2 v) N_{C C}\left(\frac{1+\theta}{\left(1-A_{0}\right)^{3 / 2}(1-R \sin \psi)^{3 / 2}}-\frac{\theta}{\left(1-A_{0}\right)^{1 / 2}(1-R \sin \psi)^{1 / 2}}\right)$.

Provided the actuation voltage $v$ is in quadrature with the displacement, taking the average of (D-4) over one period of $\psi$ yields, using (C-1) in the case $n=3 / 2$ and $n=1 / 2$ :

$\frac{2 \delta N_{C C}}{\pi}\left(\frac{1+\theta}{\left(1-A_{0}\right)^{3 / 2}} \frac{\sqrt{1+R}}{1-R^{2}} \mathrm{E}-\frac{\theta}{\left(1-A_{0}\right)^{1 / 2}} \frac{1}{\sqrt{1+R}} \mathrm{~K}\right)$.

where $\mathrm{K}$ (resp. E ) stands for the complete elliptic integral of the first (resp. second) kind, with argument $2 R /(1+R)$, as in table $\mathrm{C}-1$. 
Similarly, for a cantilever beam, the non-dimensional electrostatic force projected on the first mode may be expressed as:

$$
\delta(1+2 v) N_{C A}\left(\frac{1+\theta_{1} a}{1-a}+\left(\theta_{2}+\theta_{3} a\right) \log (1-a)\right) .
$$

Assuming (A-1) holds and making use of the reduced amplitude, (D-6) becomes:

$$
\delta(1+2 v) N_{C A}\left(\begin{array}{l}
\frac{1+\theta_{1}}{\left(1-A_{0}\right)(1-R \sin \psi)}-\theta_{1} \\
+\left(\theta_{2}+\theta_{3}\left(1-A_{0}\right)(1-R \sin \psi)\right)\left(\log \left(1-A_{0}\right)+\log (1-R \sin \psi)\right)
\end{array}\right) .
$$

Provided the actuation voltage $v$ is in quadrature with the displacement, taking the average of (D-7) over one period of $\psi$ yields, using $(\mathrm{C}-1)$ in the case $n=1,(\mathrm{C}-2)$ and $(\mathrm{C}-3)$ :

$$
\delta N_{C A}\left(\begin{array}{c}
\frac{1+\theta_{1}}{\left(1-A_{0}\right) \sqrt{1-R^{2}}}-\theta_{1}+\theta_{3}\left(1-A_{0}\right)\left(1-\sqrt{1-R^{2}}\right) \\
+\left(\theta_{2}+\theta_{3}\left(1-A_{0}\right)\right) \log \left(\frac{\left(1-A_{0}\right)}{2}\left(1+\sqrt{1-R^{2}}\right)\right)
\end{array}\right) .(\mathrm{D}-8)
$$

The expressions of $f_{\text {sin }}$ and $f_{\text {cos }}$ may be derived in a similar fashion.

\section{References}

[1] S. D. Senturia, "Microsystems Design," Kluwer Academic Publishers, Boston, 2000, pp. 249-259.

[2] P. M. Osterberg, S. D. Senturia, "M-TEST: a test chip for MEMS material property measurement using electrostatically actuated test structures", Journal of Microelectromechanical Systems, vol. 6, pp. 107-118, 1997

[3] G.N. Nielsonn G. Barbastathis, "Dynamic Pull-In of Parallel-Plate and Torsional Electrostatic MEMS Actuators", Journal of Microelectromechanical Systems, vol. 15, pp. 811-821, 2006

[4] S. Krylov, "Lyapunov Exponents as a Criterion for the Dynamic Pull-in Instability of Electrostatically Actuated Microstructures," International Journal of Non-Linear Mechanics, vol. 42, 626-642, 2007

[5] Vitaly Leus and David Elata, "On the Dynamic Response of Electrostatic MEMS Switches", Journal of Microelectromechanical Systems, vol. 17, pp. 236-243, 2008

[6] F.M. Alsaleem, M.I. Younis, L. Ruzziconi, "An Experimental and Theoretical Investigation of Dynamic Pull-In in MEMS Resonators Actuated Electrostatically", Journal of Microelectromechanical Systems, vol. 19, 794-806, 2010

[7] A. H. Nayfeh, M. I. Younis, E. M. Abdel-Rahman, "Dynamic pull-in phenomenon in MEMS resonators," Nonlinear Dynamics, vol. 48, pp. 153-163, 2007

[8] A. H. Nayfeh, M. I. Younis, "Dynamics of MEMS resonators under superharmonic and subharmonic excitations," Journal of Micromechanics and Microengineering, vol. 15, pp. 1840-1847, 2005

[9] V. Kaajakari, T. Mattila, A. Oja, H. Seppä, "Nonlinear limits for single-crystal silicon microresonators", Journal of Microelectromechanical Systems, vol. 13, pp. 715-724, 2004 
[10] G. Arndt, "System architecture and circuit design for micro and nanoresonators-based mass sensing arrays," Ph. D. thesis, Supélec, 2011

[11] A. Gelb, W. Van der Velde, "Multiple-input describing functions and nonlinear system design," McGraw-Hill, New-York, 1968

[12] T. A. Roessig; R. T. Howe, A. P. Pisano, J. H. Smith, "Surface-micromachined resonant accelerometer", International Conference on Solid State Sensors and Actuators (TRANSDUCERS), vol. 2, pp.859-862, 1997

[13] C. T. C. Nguyen, R. T. Howe, "An integrated CMOS micromechanical resonator high-Q oscillator," Journal of Solid-State Circuits, vol. 34, pp. 440-455, 1999

[14] K. E. Wojciechowski, B. E. Boser, A. P. Pisano. "A MEMS resonant strain sensor operated in air,” Micro Electro Mechanical Systems Conference (MEMS), pp. 841-845, 2004

[15] B. Bahreyni, C. Shafai, "Oscillator and frequency-shift measurement circuit topologies for micromachined resonant devices", Sensors and Actuators A, vol. 137, pp. 74-80, 2007

[16] J. Verd, et al., "Monolithic CMOS MEMS Oscillator Circuit for Sensing in the Attogram Range," Electron Device Letters, Volume.29, pp.146-148, 2008

[17] A. A. Trusov, S. A. Zotov, B. R. Simon, A. M. Shkel, "Silicon Accelerometer with Differential Frequency Modulation and Continuous Self-Calibration," Micro Electro Mechanical Systems Conference (MEMS), pp. 29-32, 2013

[18] J. I. Seeger, B. E. Boser, "Dynamics and control of parallel-plate actuators beyond the electrostatic instability," International Conference on Solid-State Sensors and Actuators (TRANSDUCERS), pp. 474-477, 1999

[19] A. Fargas-Marques, J. Casals-Terré, A. M. Shkel, "Resonant Pull-In Condition in Parallel-Plate Electrostatic Actuators," Journal of Microelectromechanical Systems, vol. 16, pp. 1044-1053, 2007

[20] J. Juillard, G. Arndt, J. Arcamone, E. Colinet, "Influence of excitation waveform and oscillator geometry on the resonant pull-in of capacitive MEMS oscillators," Symposium on Design, Test, Integration and Packaging of MEMS/MOEMS (DTIP), pp. 1-4, 2013

[21] E. Colinet, J. Juillard, S. Guessab, R. Kielbasa, "Actuation of resonant MEMS using short pulsed forces," Sensors and Actuators A, vol. 115, pp. 118-125, 2004

[22] J. Juillard, A. Bonnoit, E. Avignon, S. Hentz, and E. Colinet, "Large amplitude dynamics of micro-nanomechanical resonators actuated with electrostatic pulses," Journal of Applied Physics, vol. 107, pp. 014907-1-10, 2010

[23] J. Juillard, G. Arndt, E. Colinet, "Modeling of micromachined beams subject to nonlinear restoring or damping forces,", Journal of Microelectromechanical Systems, vol. 20, pp. 165-177, 2011

[24] http://functions.wolfram.com/HypergeometricFunctions/Hypergeometric2F1/

[25] L. D. Gabbay, J. E. Mehner, and S. D. Senturia, "Computer-aided generation of nonlinear reduced-order dynamic macromodels," Journal of Microelectromechanical Systems, vol. 9, pp. 262269,2000

[26] A. Brenes, J. Juillard, F. Vinci dos Santos, "Electrostatically induced modal crosstalk phenomena in resonant MEMS sensors", Symposium on Design, Test, Integration and Packaging of MEMS/MOEMS (DTIP), pp. 294-297, 2014 
[27] J. Juillard, "A comparative study of reduced-order modeling techniques for nonlinear MEMS beams", Symposium on Design, Test, Integration and Packaging of MEMS/MOEMS (DTIP), pp. 261265,2014 